\title{
Considerations on determining the explosion risk and the areas with explosion hazard in a natural gas compressor station
}

\author{
Alina Maria Gligor ${ }^{1, *}$, and Valentin Petrescu ${ }^{1}$ \\ ${ }^{1}$ Lucian Blaga University of Sibiu, Romania, Department of Industrial Engineering and Management, Emil Cioran str., 4, Sibiu, \\ Romania
}

\begin{abstract}
Compressor stations are important components within natural gas transportation networks. The current paper analyses some of the safety aspects involved in the operation of such facilities - on the one hand related to the explosion risk for employees working in compressor stations and on the other hand related to determining to the areas with increased explosion hazard in such a station, in order to help reducing workrelated accidents and the occurrence of professional illnesses. The explosion risk is determined based on a method devised by the National Institute for Research and Development for Environmental Protection Bucharest, while the explosion hazard areas are determined and displayed with the help of the software package AutoCAD Plant 3D.
\end{abstract}

\section{Introduction}

Natural gas is a very important energy source, but also an energy source that requires special attention with regard to the safety features of the production, storage, treatment, transportation and distribution facilities.

The assessment of explosion and fire hazards is in this case of essential importance for the selection of equipments that are to be used in such facilities and for their configuration. [1] For example, the usage of electrical equipments in areas with explosion hazard mandates an approach that differs from the case of normal areas, in several aspects.

Firstly, even before implementing any technical solution, for ensuring the explosion safety of the personnel and of the equipment operating in such demanding environments it is necessary to analyse in detail the possibility of flammable substance accumulations that may create explosive atmospheres. [2]

Secondly, it is necessary to determine the probability of an explosive atmosphere forming within the facility, taking into account and identifying all types of employed flammable substances so as to facilitate the placing of the various areas within one of the standardised types of explosion hazard areas.

While various authors have discussed the possibilities of assessing the explosion risk in various other parts of a natural gas extraction, storage and transportation system $[1,3,4]$, in the current paper, the authors have on the one hand used a consecrated method to determine the explosion risk in a natural gas compressor station and on the other hand they also employed a specialised 3D computer-aided design software, AutoCAD Plant 3D, to help indicate the main problem areas.

\section{Presentation of the analysed natural gas compressor station}

Natural gas compressor stations are facilities for the processing of natural gas coming from the extraction wells, used to increase the gas pressure in order to facilitate its transportation over large distances through main pipeline systems and for the subterraneous storage of the gases from the transportation and distribution network. [5]

In Romania, the first compressor station, with an installed capacity of $8000 \mathrm{hp}$ and used in the transportation of natural gases, started to function in the year 1965.

The compressor station chosen by the authors for the analysis comprises following elements: the installation for separating and filtering gases; the technological piping system consisting of aspiration and evacuation collector pipes, bypasses, interconnections, gas stacks, evacuators; the installation for measuring basic technological parameters (such as flows, pressures, temperatures); compressor units; cooling systems for the compressed gases, oil circuits, compressor cylinders and engines; installations for separating the oil used for lubricating the compressor cylinders; the electric energy system; compressor station rooms; systems for detecting the presence of hazardous atmospheres; fire prevention systems, systems for isolating and depressurising in the event of an accident, video surveillance systems. [5]

The analysed compressor station has been modelled using the computer-aided design software AutoCAD Plant 3D (figure 1).

\footnotetext{
* Corresponding author: alina.gligor@ulbsibiu.ro
} 


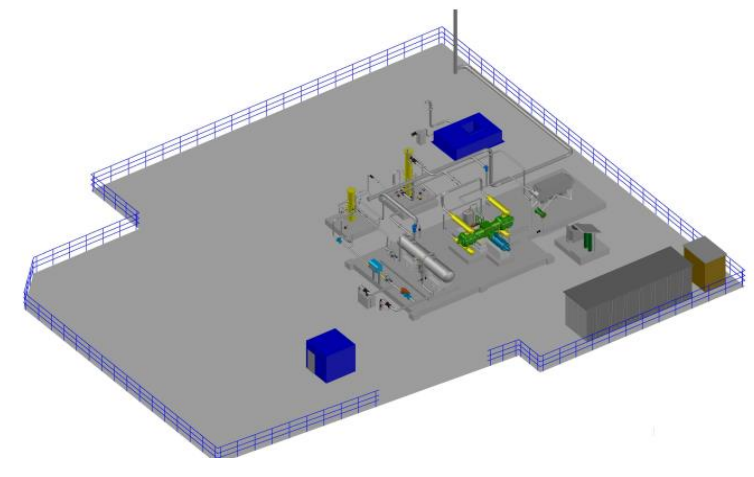

Fig.1. Schematic presentation of the analysed natural gas compressor station

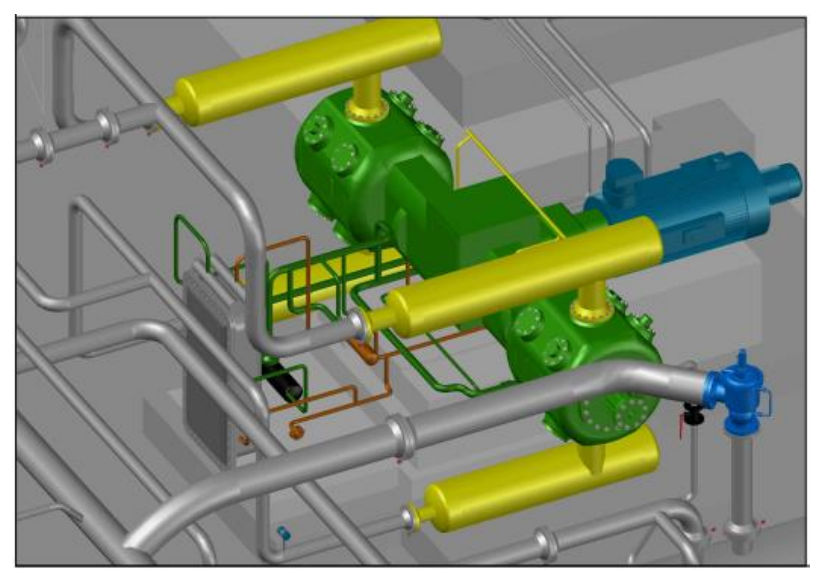

Fig. 2. Detail of a modelled equipment from the natural gas compressor station

\section{Risk Assessment Methodology}

The method employed by the author to determine the levels of explosion risk within the analysed natural gas compressor station is based on the one conceived by the National Institute for Research and Development for Environmental Protection (I.N.C.D.P.M.) Bucharest. This method involves a quantitative risk/security level determination for a workplace, area or facility, based on a systemic analysis, as well as the risk assessment for accidents and professional illnesses.

This method requires the identification of all explosion risk factors within the analysed workplace or facility, using predetermined checklists and then quantifies the risk level according to the gravity and frequency of the maximal predictable consequence. A workplace's security level is then inversely proportional to the determined risk level.

When applying the method, following steps should be followed:

- defining the analysed system (workplace);

- $\quad$ identifying the risk factors in the system;

- $\quad$ assessing the explosion risks;

- ranking the risks and determining the prevention priorities;

- $\quad$ suggesting prevention measures.

The above-mentioned steps require the employment of following work instruments:
- $\quad$ List for the identification of risk factors;

- List of possible consequences of the action of risk factors on the human body;

- Scale for ranking the gravity and probability of consequences;

- $\quad$ Risk assessment grid;

- Risk level scale and safety level scale, respectively;

- Workplace assessment form - centralising document;

- $\quad$ List of proposed measures.

A workplace's global risk level $(\mathrm{Nr})$ is then determined as weighed average of the risk levels determined for the identified risk factors. The rank of each risk factor is used as weighing element (being equal to the risk level), so that the result will reflect reality as closely as possible.

The formula for calculating the global risk level is given in (1):

$$
N_{r}=\frac{\sum_{i=1}^{n} r_{i} * R_{i}}{\sum_{i=1}^{n} r_{i}}
$$

where:

- $\mathrm{N}_{\mathrm{r}}$ is the global risk level for a workplace;

- $r_{i}$ is the rank of the risk factor ,i”;

- $\mathrm{R}_{\mathrm{i}}$ is the risk level for the risk factor , $\mathrm{i}$ ";

- $\mathrm{n}$ is the number of risk factors identified for the considered workplace.

\section{Assessing the explosion risk in the analysed natural gas compressor station}

In the case of the natural gas compressor station, the explosion risk factors were determined taking into account the tasks indicated in the job description files for the personnel operating within this facility, as well as the corresponding technological processes. The risk factors were then identified and grouped by the generating element within the facility (operator, work task, production means and work environment).

For the next step, the authors employed the previously mentioned work instruments in order to determine the partial risk level for each risk factor.

The global risk level for the workplace "Natural gas compressor station" has been calculated using formula (1) and the partial risk levels indicated in figure 3 , leading to value of $\mathrm{N}_{\mathrm{r}}=2.58$.

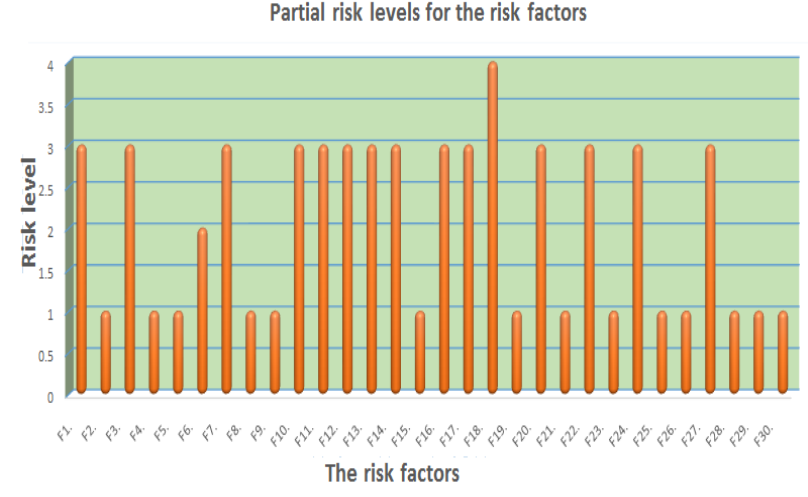

Fig. 3. Partial risk levels for the risk factors at the natural gas compressor station 


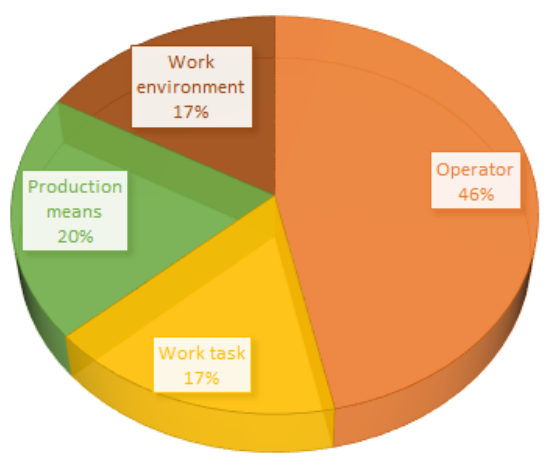

Fig.4. Distribution of the risk factors by generating sources

Table 1 presents some examples of risk factors, corresponding to the 4 types of explosion risk generating sources specific for the natural gas compressor station.

Table 1. Examples of risk factors taken into account

\begin{tabular}{|c|c|}
\hline $\begin{array}{l}\text { Risk factor } \\
\text { generating } \\
\text { source }\end{array}$ & Risk factors \\
\hline \multirow[t]{2}{*}{ Operator } & $\begin{array}{l}\text { F1. Carrying out tasks not included in } \\
\text { the job requirements }\end{array}$ \\
\hline & $\begin{array}{l}\text { F11. Entering the compressor area } \\
\text { without adequate protection equipment, } \\
\text { or with equipment that can lead to } \\
\text { electrostatic discharges }\end{array}$ \\
\hline \multirow[t]{2}{*}{ Work task } & $\begin{array}{l}\text { F17. Wrong succession of operations or } \\
\text { absence of operations required by } \\
\text { technological documents }\end{array}$ \\
\hline & $\begin{array}{l}\text { F18. Employment of personnel who do } \\
\text { not have the necessary competence or } \\
\text { qualification for the assigned work task }\end{array}$ \\
\hline \multirow[t]{2}{*}{$\begin{array}{c}\text { Work } \\
\text { equipment }\end{array}$} & $\begin{array}{l}\text { F24. Contact with moving elements of } \\
\text { the compressor }\end{array}$ \\
\hline & $\begin{array}{l}\text { F25. Lack of equipotential and grounding } \\
\text { connections on the instruments or on other } \\
\text { electrical equipments }\end{array}$ \\
\hline \multirow[t]{2}{*}{$\begin{array}{c}\text { Work } \\
\text { environment }\end{array}$} & $\begin{array}{l}\text { F26. Loud noise level (above the } \\
\text { maximally admitted level) near the } \\
\text { compressor and during the opening of } \\
\text { the pressure relief valves }\end{array}$ \\
\hline & $\begin{array}{l}\text { F30. Inadequate lighting level during works } \\
\text { carried out during the evening or night }\end{array}$ \\
\hline
\end{tabular}

Thus, the global risk level calculated for the workplace "Natural gas compressor station engineer" is 2.58 , a value that places it among the workplaces with small to medium risk levels.

From figure 2 it can be noticed that of the total of 30 identified risk factors, only 1 (F18) surpasses a partial risk level of 3 , thus being considered medium to high risk factors.

By determining the explosion risk level, it is possible to determine the priorities in prevention measures for the workplace, but also to optimise the usage of resources allocated for this purpose.

The explosion risk in installations where explosive atmospheres may form can be reduced in two ways:
- continuous checking of the concentration of flammable in the area of equipments and initiating their decoupling from the electric power network if approaching the explosion limit;

- construction and usage of electric equipment with specific constructive and functional characteristics, that cannot become ignition sources for the explosive atmosphere that surrounds them.

An advantage of the I.N.C.D.P.M. method is the fact that it does not require an assessed system to be physically present. Also, it can be employed for all stages of a system's (or of an element of this system's) lifecycle: conception and design, physical creation, production, actual functioning, dismantling.

\section{Indication of the explosion risk areas}

An explosion risk area represents an industrial space in which, under normal functioning conditions, there can accumulate, permanently or accidentally, gases, vapors of flammable liquids, dusts or powders in sufficient amounts to give birth to an explosive atmosphere. Explosion risk areas are consequently very important from the point of view of the work safety legislation $[7,8]$ and require a special training of the personnel working within or near them. [9]

Explosion hazard areas can be classified into several types, function of the length of the periods during which the explosive atmosphere is present: $[5,10]$

- area 0 - is the area in which the explosive atmosphere is present during the normal functioning of the technological installations, permanently or for a period of more than 1000 hours per year.

- area 1 - is the area in which the explosive atmosphere can occur during the normal functioning of the technological installations for intermittent periods with durations comprised between 10 and 1000 hours per year.

- area 2 - comprises the industrial areas within which an explosive atmosphere cannot occur during the normal functioning, but only in cases of predictable defects or for periods between 0.1 and 10 hours per year.

The zoning of industrial spaces represents the process of classification of spaces within an industrial installation function of the probability of occurrence of an explosive atmosphere and is important for the subsequent correct selection of the electrical and mechanical equipment that will be used in that area.

An area's extent depends mainly on the physical and chemical parameters of the flammable material and of the production process, such as:

- Discharge flow of gases and vapours

- The lower explosion limit (LEL)

- Amount of ventilation

- Relative density of the gas or of the vapours at the moment of discharge

- Climatic conditions

- Local topography

The result of the zoning process is a set of documents that comprises maps with precise indications regarding the different areas within facilities, to which there are 
associated specific parameters for the explosion hazard (explosion group and subgroup, temperature class, area index etc).

The electrical apparatuses for normal environments cannot be used, in general, in areas with explosion hazard due to the risk of the explosive atmosphere igniting due to electric sparks or arcs, hot surfaces of equipments, electrostatic discharges etc. [5]

Therefore, at the execution of the electrical apparatuses and equipments for potentially explosive areas, there have to be applied certain supplemental constructive measures that would offer them safety during functioning and in the presence of the explosive atmosphere.

For the zoning of the natural gas regulating and metering station, the author has used the software package AutoCAD Plant 3D, that allowed to clearly emphasise the areas with explosion hazard, as shown in figure 5 .

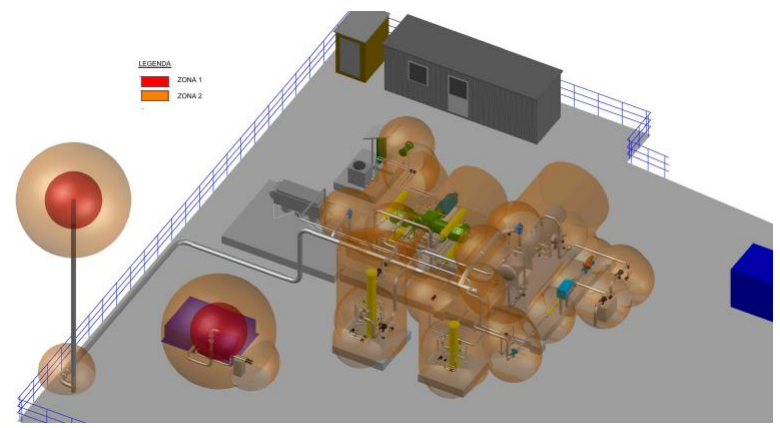

Fig. 5. 3D zoning plan showing the physical location of areas with explosion hazard

The computer-aided zoning allowed to notice that for the analysed natural gas regulating and metering station, area 1 is found for the following equipments: odorisation room, exhauster, safety valve, inner technological installation, while area 2 is encountered for the outer technological installation.

Figure 6 shows the extent of the explosion hazard areas in vertical cross-section.

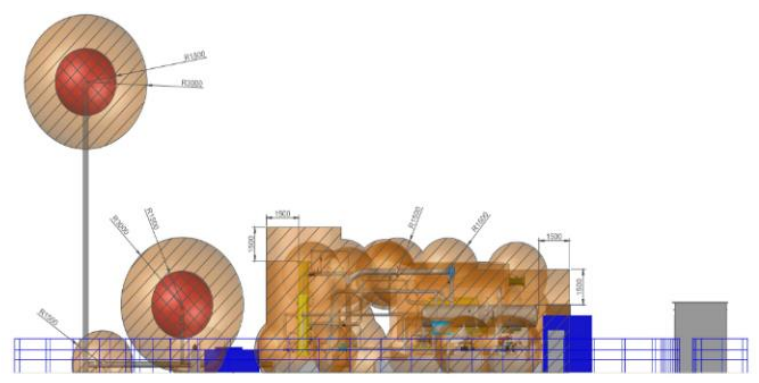

Fig. 6. Vertical zoning plan showing the physical location of the areas with explosion hazard

\section{Conclusions}

One of the main advantages of the method presented in this paper is that its application is not limited by the condition of a physical existence of the system to be assessed. It can be used in all stages related to the lifecycle of a work system or of an element of such a system: conceiving and design, physical creation, putting into production, unfolding of the work processes.

The application of the gravity/probability method for the analysed workplace has allowed the identification of explosion risk factors and to determine the dimension of risks for the occurrence of an explosion at this workplace.

For this, for each identified risk factor there will be indicated technical and organisational measures that need to be taken in order to keep the risk level in the ,area of acceptable risks".

After the division of the space into specific hazard areas, the electrical installations can be conceived either through the usage of electrical equipments certified for such areas, or, if no such equipment is available, through conceiving new equipments and the certifying it.

In order for an explosion to happen, it is necessary that an explosive atmosphere and an ignition source coexist. Therefore, in order to reduce the explosion hazard, there need to be taken measures for the prevention of explosions, by:

- avoiding explosive atmospheres. This goal can be achieved mainly by modifying either the concentration of the flammable substance to a value outside of the explosion range or the oxygen concentration to a value below the limit oxygen concentration (LOC);

- avoiding all possible ignition sources;

As a general rule, the equipments with potential ignition sources have to be, if at all possible, placed in non-hazardous areas or, if that is not possible in the least hazardous area.

\section{References}

1. D.P. Nolan, Handbook of fire and explosion protection engineering principles for oil, gas, chemical and related facilities (Elsevier, 2014)

2. D.M. Duse, C.S. Duse, C. Deac, Wiert Naft Gaz, 25, $2(2008)$

3. Y.-D. Jo, D.A. Crowl, J Loss Prevent Proc, 21, 8 (2008)

4. Moraru, R.I., Proc. 18th Conf. Envir. Min Proc., 2014

5. A.-S. Huidan, Electrical equipment for automation and control in environments with explosion hazard (Translation from Romanian) (Technical Publishing House, 2008)

6. I. Foidas, Testing and production of natural gas deposits (Translation from Romanian) (Publishing House of the „Lucian Blaga” University of Sibiu, 2014)

7. L. Tarnu, Elements of Law and Legislation (Translation from Romanian) (Publishing House of the „Lucian Blaga” University of Sibiu, 2014)

8. L. Tarnu, International protection of human rights (Translation from Romanian) (Tehnomedia Publishing House, 2014)

9. D.M. Duse, C.S. Duse, C. Deac, M.I. Rusu, Trans Adv Eng Edu, 3, 9 (2006)

10. ASRO, Explosive atmospheres. Part 10-1: Classification of hazardous areas (ASRO, 2008) 